\title{
Uso dos Serviços Públicos de Saúde para DST/ HIV/aids por Comunidades Remanescentes de Quilombos no Brasil
}

\section{Use of STD/HIV/AIDS-Oriented Public Health care Services by Quilombo Communities in Brazil}

\author{
Maria Josenilda Gonçalves da Silva \\ Mestre em Saúde Pública. Docente da Escola Superior em Ciências \\ da Saúde. Núcleo de Estudos de Saúde Pública (NESP), Centro de \\ Estudos Avançados Multidisciplinares (CEAM), Universidade de \\ Brasília, Brasília, DF, Brasil. \\ Endereço: Núcleo de Estudos de Saúde Pública, NESP. Endereço: \\ Universidade de Brasília, SCLN 407 Bloco A, sala 222, Asa norte, \\ Brasília, DF, Brasil. \\ E-mail: maria.josenilda®yahoo.com.br \\ Francisca Sueli da Silva Lima \\ Mestre em Ciências da Saúde. Docente da Escola Superior em \\ Ciências da Saúde Núcleo de Estudos de Saúde Pública (NESP), \\ Centro de Estudos Avançados Multidisciplinares (CEAM), Univer- \\ sidade de Brasília. \\ Endereço: Núcleo de Estudos de Saúde Pública, NESP. Universi- \\ dade de Brasília, SCLN 407 Bloco A, sala 222, Asa norte, Brasília, \\ DF, Brasil. \\ E-mail: suelisabino_carneiroळyahoo.com.br

\section{Edgar Merchan Hamann} \\ Doutor Saúde Pública Professor Adjunto da Universidade de \\ Brasília Núcleo de Estudos de Saúde Pública (NESP), Centro de \\ Estudos Avançados Multidisciplinares (CEAM), Universidade de \\ Brasília.Endereço: Núcleo de Estudos de Saúde Pública, NESP. \\ Universidade de Brasília, SCLN 407 Bloco A, sala 222, Asa norte, \\ Brasília, DF, Brasil. \\ E-mail: hamannœunb.br
}

\section{Resumo}

Introdução: A epidemia de aids atinge com maior intensidade os grupos mais vulneráveis. As tendências de interiorização, pauperização e feminização demonstram que a população negra se encontra em desvantagem social no que se refere à construção de respostas de enfrentamento. Objetivo: Descrever as condições de acesso da população negra ao diagnóstico e à assistência para DST, HIV/aids. Métodos: Estudo transversal com 218 sujeitos maiores de 18 anos com vida sexual ativa em 11 comunidades quilombolas. Resultados: $75 \%$ utilizam os serviços públicos na atenção básica. Antecedentes de sinais e sintomas de DST foram relatados por $10 \%$ dos entrevistados e a maioria procurou o serviço público de saúde. A testagem para HIV foi realizada por $22 \%$. 0 serviço público não especializado foi utilizado por $73 \%$. As mulheres que referiram sinais e sintomas de DST procuraram o serviço público e realizaram o teste para HIV com maior frequência que os homens. Houve uma maior percepção de dificuldades de atendimento, busca de assistência no serviço privado e testagem mais frequente entre os mais jovens. Pessoas negras perceberam maior dificuldade no atendimento, maior relato de sinais e sintomas de DST e maior frequência de automedicação quando comparadas às não negras. Contudo, a avaliação do serviço foi considerada ótima/boa por $45 \%$. Entre pessoas não negras houve maior procura pelo serviço público. Conclusão: 0 estudo reafirma a necessidade de políticas públicas voltadas aos segmentos mais vulneráveis. É importante ressaltar a necessidade da capacitação das equipes do PACS (Programa de Agentes Comunitários de Saúde) e PSF (Programa Saúde da Família) pela sua relevância na assistência dessas comunidades.

Palavras-chave: DST; HIV; Aids; População negra; Remanescente de quilombos. 


\section{Abstract}

Introduction: The AIDS epidemic affects vulnerable groups with more intensity. Tendencies show that countryside territories, poor populations and female patients are vulnerable; thus, the black population find themselves in social disadvantage regarding the construction of coping responses. Objectives: To describe the black population's conditions of access to HIV/AIDS and STD diagnosis and assistance, and the use of healthcare services in such context. Methods: Cross-sectional epidemiological study with a sample of 218 sexually active adults, living in 11 different quilombos (hiding places of runaway slaves) in Brazil. Results: $75 \%$ said they use public primary care services. Previous symptoms of STD were reported by $10 \%$ of the interviewees, and most of them said they contacted the public healthcare service $(70 \%)$. HIV testing was performed by $22 \%$ of the subjects. The public non-specialized healthcare service was used by $73 \%$ of the interviewees. Among the participants who reported previous symptons of STD, women went to the public healthcare service and performed the HIV testing with higher frequency than men. Young people had greater perception of assistance difficulties, and they also searched more for assistance in the private healthcare services and performed testing more frequently. Black people perceived greater difficulty in receiving assistance, reported previous symptoms of STD with higher frequency, and resorted to self-medication more often when compared to non-black individuals. However, service ratings were considered good/great by $45 \%$ of the participants. Non-black people claimed to seek for public services more frequently than the other subjects. Conclusion: The study highlights the necessity of public policies targeted at the most vulnerable segments of population. It is important to mention the need to train the medical teams of Programa de Agentes Comunitários de Saúde (PACS - Health Community Agents Program) and Programa Saúde da Família (PSF - Family Health Program), due to their relevance in the assistance provided for these communities.

Keywords: STD; HIV; AIDS; Black Population; Quilombo Population.

\section{Introdução}

Nos últimos anos, vem aumentando consideravelmente no país o número de pesquisas sobre as questões de identidade étnico-raciais visando o acesso a ações e serviços de saúde. Entretanto, apesar dos avanços, ainda existem lacunas que precisam ser entendidas, daí a importância de estudos que utilizem o quesito cor/raça e possibilitem uma análise mais detalhada desta questão (Simão e col., 2007).

Os quilombos brasileiros e as áreas remanescentes são fruto de um processo histórico da formação da nação brasileira, originada na colonização portuguesa. A formação dos quilombos se deu como forma de resistência ao regime escravocrata durante o Brasil Colônia e ainda no Império, sendo que até os dias atuais são ocupados por populações sem o integral reconhecimento da sociedade. 0 fenômeno dos quilombos não é apenas um evento da realidade brasileira. Com nomes distintos, em toda a América, onde houve escravidão, houve resistência e a formação dessas comunidades, como os palenques ou cumbés na América espanhola, os maroons e grand marronage nas áreas de colonização inglesa e francesa, respectivamente. Em nosso país, o aquilombamento tornou-se símbolo da resistência e tem em Zumbi dos Palmares o seu máximo expoente (Gomes, 2005).

Muito recentemente, os ocupantes de áreas de quilombo e de espaços outorgados ou ocupados no momento da emancipação obtiveram reconhecimento do Estado sobre as terras. O Decreto 4.887, de 20 de novembro de 2003 , em seu artigo $2^{\circ}$, considera os remanescentes das comunidades dos quilombos como grupos étnico-raciais, segundo critérios de autoatribuição, com trajetória histórica própria, dotados de relações territoriais específicas, com presunção de ancestralidade negra, relacionada com a resistência à opressão histórica sofrida. A partir desse decreto, fica a cargo do Ministério do Desenvolvimento Agrário, por meio do Instituto Nacional de Colonização e Reforma Agrária (Incra), identificação, reconhecimento, delimitação, demarcação e titulação das terras ocupadas pelos remanescentes das comunidades dos quilombos.

Para fins de definição das comunidades e áreas, a Fundação Cultural Palmares fica responsável, 
entre outras funções, pela inscrição dos quilombos no Cadastro Nacional e pela expedição de certidão. Em mais de quatro anos, foram emitidas aproximadamente 960 certidões, comportando mais de 1171 comunidades certificadas, já que em algumas certidões constam mais de uma comunidade.

A certificação por parte da Fundação Cultural Palmares tem sido de fundamental importância para as comunidades remanescentes de quilombos, pois, se em seus aspectos físicos e materiais, não houve grandes alterações, apesar de aparentes melhoras, no que se diz respeito à busca por uma reconstrução de identidades negras e ao redespertar do orgulho do seu passado, vem trazendo mudanças significativas no próprio olhar dos componentes dessas comunidades sobre si mesmos. Como consequência disso, e por políticas setoriais paralelas de ação afirmativa, o olhar das comunidades externas aos quilombolas tem passado por mudanças, atingindo também os governos estaduais e municipais.

Apesar dos avanços, as populações remanescentes de quilombos ainda sofrem uma diversidade de problemas, desde disputas agrárias em torno das áreas ocupadas, até a falta de infraestrutura e investimentos públicos por parte do Estado. A ausência de ensino, a ainda precária presença de serviços de saúde, o isolamento físico e social dessas comunidades, o preconceito e a falta de informação, entre outros elementos, têm perpetuado uma série de distorções acerca do conhecimento sobre a saúde e sobre o alcance de políticas de promoção e prevenção.

Com relação às DST e HIV/aids, a informação é a ferramenta fundamental para a prevenção e o enfrentamento, o que torna de fundamental importância buscar conhecer qual o nível de conhecimento sobre o assunto entre essa população, para, a partir daí, buscar soluções e políticas públicas.

A Política Nacional de Saúde Integral da População Negra aprovada em novembro de 2006 pelo Conselho Nacional de Saúde (CNS) propõe ações transversais inseridas no Sistema Único de Saúde (SUS), visando garantir a efetivação do direito à saúde da população negra em relação a promoção, prevenção e tratamento dos agravos transmissíveis e não transmissíveis (Brasil, 2006).

Estudos dão conta de que a epidemia de aids atinge com maior intensidade os grupos mais vulneráveis, ou seja, os grupos histórica e culturalmente discriminados ou excluídos (Lopes, 2003). Um aspecto relevante da vulnerabilidade à infecção com o vírus HIV está vinculado ao acesso a serviços de saúde com qualidade e resolubilidade (Mann e Tarantola, 1996; Ayres, 2003).

Em relação à epidemia do HIV/aids, as tendências de interiorização, pauperização e feminização fazem com que populações antes não atingidas, como a população negra remanescente de quilombos, se deparem com condições e situações de desvantagem social no que se refere à construção de respostas de combate à epidemia, por estarem à margem da maioria das políticas públicas de saúde (Brasil, 2005). Historicamente, esse segmento da população se torna mais vulnerável pelas dificuldades de acesso às ações de saúde, bem como à informação sobre a epidemia (Lopes, 2003).

Desde 2000, o Departamento de DST, Aids e Hepatites Virais do Ministério da Saúde vem disponibilizando dados desagregados por cor/raça e esse registro vem melhorando a cada ano. Em 200o, em cerca de $48 \%$ dos casos de aids notificados no Sistema de Informação de Agravos de Notificação (Sinan), essa variável não era registrada. Esse percentual caiu para $6,7 \%$ em 2008, à época de realização da pesquisa. A proporção relatada como sendo de cor branca foi predominante em 2008 (56\%), seguida da cor parda (31\%), preta ( $12 \%)$, sendo a maior discrepância entre homens e mulheres registrada nessa última categoria ( $9,8 \%$ dos homens e $13,9 \%$ das mulheres notificados). As cores/etnia amarela ou indígena são registradas com menores frequências (menos de 1\% cada uma) (Brasil, 2005).

Este artigo tem como objetivo descrever as condições de acesso e uso dos serviços de saúde por parte dos residentes de comunidades remanescentes de quilombos para o diagnóstico de HIV e assistência às DST.

\section{Métodos}

Foi realizada uma pesquisa qualiquantitativa que compreende um componente epidemiológico do tipo transversal e um componente qualitativo. 0 estudo foi realizado de janeiro de 2007 a janeiro de 2008.

Foram selecionadas, de forma aleatória, 12 comu- 
nidades remanescentes de quilombos, uma por estado, já certificadas pela Fundação Palmares ou em processo de certificação. A participação dos agentes comunitários de saúde e de lideranças comunitárias locais foi a estratégia adotada para acessar a população de estudo em suas comunidades, já que se tratava de área com especificidades sociais. 0 trabalho de campo foi realizado pelos próprios pesquisadores, que desempenharam a função de entrevistador e coordenador de campo, acompanhados por estagiários treinados para a função de entrevistador.

No geral, a equipe foi muito bem recebida pelos moradores das comunidades. 0 contato prévio com as lideranças facilitou o acesso às comunidades.

Foram incluídos no estudo maiores de 18 anos com vida sexual ativa referida que aceitaram ser entrevistados e assinaram um Termo de Consentimento Livre e Esclarecido. Um total de 218 entrevistados respondeu voluntariamente a um questionário semiestruturado, previamente validado em um estudo-piloto realizado junto a uma comunidade quilombola de Goiás, próxima do Distrito Federal.

O questionário semiestruturado com 70 questões abrangeu as seguintes áreas: dados sociodemográficos, comportamento e práticas sexuais, acesso aos serviços de saúde, sinais e sintomas compatíveis com DST, uso dos serviços de saúde para diagnóstico e tratamento de DST, testagem de HIV e grau de satisfação com os serviços.

Para o componente qualitativo do estudo, foram realizados grupos focais, que buscaram aprofundar as motivações e percepções relacionadas com as variáveis mais importantes do estudo seguindo um roteiro. Por tratar-se de um artigo descritivo, quantitativo, as falas dos participantes dos grupos focais são meramente ilustrativas, em relação ao acesso aos serviços básicos de saúde.

Para efeitos da descrição do acesso e utilização dos serviços de saúde, no componente quantitativo, foram apuradas as prevalências e realizadas análises comparativas por gênero, idade (com ponto de corte em 35 anos), e cor autorreferida recategorizada. 0 quesito "cor da pele" foi autorreferido, tendo sido empregadas as categorias utilizadas pelo Instituto Brasileiro de Geografia e Estatística (IBGE, 200o). Para efeito de análise, optou-se por utilizar as categorias: "pessoas negras" e "pessoas não negras", sendo o grupo de "negras" composto de participantes que se autodeclararam negros ou pretos, e o de "não negras", a soma das demais categorias, inclusive pardos. As variáveis foram categorizadas levando-se em conta as medianas das medidas e, no caso do item renda, foram realizadas estratificações baseadas no valor do salário mínimo da época da pesquisa.

A inserção dos questionários no banco de dados e as tabulações foram feitas mediante a utilização do software Epi-info 3.3.2/2005.

$\mathrm{Na}$ análise dos dados qualitativos foram incluídas a leitura flutuante dos dados transcritos e a identificação de ideias centrais e de categorias de ancoragem presentes nas falas.

O estudo foi elaborado em consonância com a legislação que regulamenta a pesquisa com seres humanos no país, Resolução 196/96 do Conselho Nacional de Saúde (CNS). 0 protocolo foi submetido e aprovado pelo Comitê de Ética em Pesquisa da Secretaria de Saúde do Distrito Federal, parecer $N^{\circ}$ 035/2007. A participação no estudo foi voluntária e anônima.

\section{Resultados}

Os 218 entrevistados, tendo como critério de inclusão no estudo a residência na comunidade, estavam distribuídos em 11 comunidades remanescentes de quilombos das cinco regiões do país: Boqueirão, Vitória da Conquista (Bahia), Conceição dos Caetanos, Tururu (Ceará), Magalhães, Nova Roma (Goiás), Felipa, Itapecurumirim (Maranhão), Mata Cavalo, Livramento (Mato Grosso), Brejo dos Crioulos, Vazerlândia (Minas Gerais), Baixo Ipixúna, Gurupá (Pará), Sumidouro, Queimada Nova (Piauí), Mormaça, Sertão (Rio Grande do Sul), Boa Vista dos Negros, Parelhas (Rio Grande do Norte), e André Lopes, Registro (São Paulo). Os moradores da comunidade Serrote do Gado Brabo, localizada no município de São Bento do Una, em Pernambuco, recusaram-se a participar do estudo por problemas de articulação interna e falta de consenso.

Do total, apenas $68,1 \%$ dos participantes se autodefiniram "negros". A tabela 1 apresenta a distribuição percentual da população do estudo segundo variáveis socioeconômicas e demográficas. A amostra foi constituída, na sua maioria, por 
mulheres, correspondendo a cerca de 58\%. Quanto à idade, houve variação de 18 e 81 anos (média = 36 anos; mediana 33; moda 18).

Os entrevistados constituíam um grupo de pessoas com nível de escolaridade baixo: $29,5 \%$ nunca frequentaram a escola e apenas $6 \%$ concluíram o ensino fundamental. Destaca-se que cerca de $3 \%$ dessa população sabia ler apesar de nunca terem ido à escola; apenas $2,3 \%$ cursavam o ensino superior.

No quesito ocupação, 21\% dos participantes eram trabalhadores rurais temporários e $10 \%$ estavam desempregados. Nessa amostra, embora esteja representado um percentual expressivo de jovens, apenas 1,6\% referiram ser estudantes. Com relação à renda 57\%, aproximadamente, informaram obter rendimentos, variando entre R\$ 50,0o e R\$ 80o,oo ( média = R\$238,oo; mediana = R\$200,oo).

Quanto ao estado civil, predominou a categoria casado (51\%); somado-se estes aos que referiram morar juntos (16,5\%), obteve-se um total de $67,4 \%$ dos entrevistados. Os solteiros correspondiam a cerca de $26 \%$ dos entrevistados.

Quanto ao uso dos serviços de saúde (Tabela 2), a maioria (cerca de 75\%) utilizava os serviços públicos no nível da atenção básica. Destaca-se que mais de 30\% dos municípios tinham implantado um dos programas que envolvem a visita domiciliar do Agente Comunitário de Saúde (ACS): o Programa de Agentes Comunitários de Saúde (PACS) ou o Programa de Saúde da Família (PSF, com equipes multiprofissionais). É interessante observar que cerca de $\mathbf{2 0} \%$ mencionaram o acesso ao sistema via hospital. O acesso, em geral, foi percebido como difícil, conforme expresso a seguir:

Se não tiver dinheiro para fretar um carro pra levar, se for depender de ambulância, quando ver é o carro da funerária. (Grupo focal - feminino - Conceição dos Caetanos - Água Preta

- Tururu,CE.).

A distância da residência ao serviço de saúde variou muito. Em geral, o serviço era distante por tratar-se de comunidades rurais. A maioria se deslocava a pé ao serviço de saúde (38\%). Ônibus e carros foram utilizados por cerca de uma quarta parte dos entrevistados ( $25 \%$ e $24 \%$, respectivamente). Moto- cicleta e bicicleta foram também utilizados (14\% e $10 \%$, respectivamente). Outros meios de transporte foram referidos com menor frequência (barco, voadeira, dentre outros), correspondendo a 10\%. 0 grau de dificuldade estava relacionado à disponibilidade de veículos, acesso de ambulâncias ao local do quilombo, à dificuldade ou impossibilidade de transportar doentes e/ou feridos.

Mais da metade dos entrevistados mencionou dificuldades no atendimento pelo sistema público de saúde. As dificuldades, para a maioria dos respondentes, ligavam a distância da residência aos horários de atendimento, longas filas e outras barreiras.

Quando precisam eles encaminham pra esses outros locais, porque o hospital da cidade não tem capacidade pra fazer tratamento. $O$ teste faz aqui, mas tratamento não. (Grupo focal - feminino - Boa Vista dos Negros, RN.).

Antecedentes de sinais e sintomas de DST nos últimos seis meses foram relatados por cerca de $10 \%$ dos entrevistados. É interessante constatar que a maioria procurou o serviço público de saúde (70\%). Preocupa o fato de $10 \%$ não ter tomado nenhuma providência apesar de parte dessa demanda poder corresponder a uretrites ou vaginites inespecíficas.

A testagem para HIV foi realizada por cerca de 22\% dos participantes. O serviço público de saúde não especializado foi o local mais utilizado (73\%) e houve utilização de serviços especializados (11\%). Houve também uso dos bancos de sangue, embora não tenha sido determinado com clareza se as pessoas procuraram esse serviço apenas para realizar o teste, como se verificou em estudos anteriores. Os serviços privados foram procurados por cerca de $7 \%$ dos que realizaram o teste. Em geral, o número de testes sorológicos realizados variou de um a cinco (média $=1,9$; mediana $=2$ ). A grande maioria dos indivíduos que fizeram o teste (98\%) procurou o resultado do teste.

Quanto à distribuição das variáveis de utilização dos serviços segundo gênero (tabela 3), destaca-se que as mulheres que referiram sintomas e sinais de DST procuraram o serviço público de saúde e realizaram o teste para HIV com maior frequência quando comparadas com os homens. 
Tabela I - Distribuição da população remanescente de quilombos, segundo características, socioeconômicas e demográficas. Brasil, 2007-2008

\begin{tabular}{|c|c|c|c|}
\hline & & Frequência & Percentual \\
\hline \multirow{3}{*}{ Sexo } & Feminino & 121 & 55,5 \\
\hline & Masculino & 97 & 44,5 \\
\hline & Total & 218 & 100,0 \\
\hline \multirow{7}{*}{ Faixa Etária } & $18-24$ anos & 55 & 25,2 \\
\hline & $25-34$ anos & 61 & 28,0 \\
\hline & $35-44$ anos & 39 & 17,9 \\
\hline & $45-54$ anos & 34 & 15,6 \\
\hline & $55-64$ anos & 21 & 9,6 \\
\hline & 65 e mais & 08 & 3,7 \\
\hline & Total & 218 & 100 \\
\hline \multirow{5}{*}{ Cor autorreferida } & Branca & 10 & 4.7 \\
\hline & Negra & 145 & 66.8 \\
\hline & Parda & 61 & 27.10 \\
\hline & Não sabe & ol & 0,7 \\
\hline & Total & 218 & 100,0 \\
\hline \multirow{7}{*}{ Escolaridade } & Nunca estudou & 51 & 23,5 \\
\hline & Fundamental incompleto & 109 & 50,2 \\
\hline & Fundamental & 13 & 6,0 \\
\hline & Médio incompleto & 15 & 6,9 \\
\hline & Médio & 24 & 11,1 \\
\hline & Superior incompleto & 05 & 2,3 \\
\hline & Total & 217 & 100,0 \\
\hline \multirow{6}{*}{ Estado Civil } & Casado & III & 50,9 \\
\hline & Solteiro & 56 & 25,7 \\
\hline & Viúvo & 08 & 3,7 \\
\hline & Divorciado & 07 & 3,2 \\
\hline & Outros & 36 & 16,5 \\
\hline & Total & 218 & 100,0 \\
\hline \multirow{9}{*}{ Sit. trabalho } & Emprego fixo & 22 & 11,8 \\
\hline & Sem vínculo (bico) & 27 & 14,5 \\
\hline & Boia-fria & 43 & 23,2 \\
\hline & Desempregado & 19 & 10,2 \\
\hline & Aposentado & 18 & 9,7 \\
\hline & Estudante & 3 & 1,6 \\
\hline & Dona de casa & 19 & 10,2 \\
\hline & Outros & 35 & 18,8 \\
\hline & Total & 186 & 100,0 \\
\hline \multirow{8}{*}{ Renda pessoal ( $R \$$ ) } & Sem renda & 95 & 43,6 \\
\hline & Até 50 & 08 & 3,7 \\
\hline & 51 a 100 & 38 & 17,4 \\
\hline & 101 a 200 & 22 & 10,1 \\
\hline & 201 a 380 & 44 & 20,2 \\
\hline & 381 a 570 & 05 & 2,3 \\
\hline & 571 a 1140 & 06 & 2,8 \\
\hline & Total & 218 & 100,0 \\
\hline
\end{tabular}


Tabela 2 - Acesso aos serviços públicos de saúde da população remanescente de quilombos, Brasil, 2007-2008

\begin{tabular}{|c|c|c|c|}
\hline & & Frequência & Percentual \\
\hline \multirow{6}{*}{$\begin{array}{l}\text { Serviço público mais } \\
\text { disponível na comunidade }\end{array}$} & Centro de saúde da SMS* & 91 & 44,8 \\
\hline & Unidade de saúde do PACS** & 19 & 9,4 \\
\hline & Unidade de saúde do PSF** & 44 & 21,7 \\
\hline & Hospital público & 38 & 18,7 \\
\hline & Outros & II & 5,4 \\
\hline & Total & 203 & 100,0 \\
\hline \multirow{3}{*}{$\begin{array}{l}\text { Percepção de dificuldade de } \\
\text { atendimento }\end{array}$} & Sim & 108 & 52,7 \\
\hline & Não & 97 & 47,3 \\
\hline & Total & 205 & 100,0 \\
\hline \multirow{3}{*}{$\begin{array}{l}\text { Antecedente de feridas/ } \\
\text { corrimento }\end{array}$} & Sim & 20 & 9,3 \\
\hline & Não & 194 & 90,7 \\
\hline & Total & 214 & 100,0 \\
\hline \multirow{5}{*}{$\begin{array}{l}\text { Providências tomadas } \\
\text { diante desses sintomas e } \\
\text { sinais }\end{array}$} & Busca de farmácia na comunidade & 1 & 5,0 \\
\hline & Busca de serviço público de saúde & 14 & 70,0 \\
\hline & Não fez nada & 2 & 10,0 \\
\hline & Outros & 3 & 15,0 \\
\hline & Total & 20 & 100,0 \\
\hline \multirow{3}{*}{$\begin{array}{l}\text { Antecedente } \\
\text { de testagem para HIV }\end{array}$} & $\operatorname{sim}$ & 47 & 21,7 \\
\hline & Não & 170 & 78,3 \\
\hline & Total & 217 & 100,0 \\
\hline \multirow{5}{*}{$\begin{array}{l}\text { Local onde fez o teste } \\
\text { sorológico }\end{array}$} & Serviço público (geral) & 33 & 73,3 \\
\hline & Serviço público (especializado) & 5 & $\mathrm{II}, \mathrm{I}$ \\
\hline & Serviço privado & 3 & 6,7 \\
\hline & Banco de sangue / hemocentro & 4 & 8,9 \\
\hline & Total $^{*}$ & 45 & 100,0 \\
\hline
\end{tabular}

* Secretaria Municipal de Saúde local.

** Programa de Agentes Comunitários de Saúde.

*** Programa de Saúde da Família.

Em relação à distribuição das variáveis de utilização dos serviços segundo idade (maior ou menor de 35 anos - Tabela 4), os dados apontam para uma maior percepção de dificuldades de atendimento, relato de sinais e sintomas recentes de DST, busca de assistência para DST no serviço privado e testagem mais frequentes entre os participantes mais jovens. Já o conhecimento sobre onde fazer o teste de HIV foi maior entre as pessoas com mais de 35 anos.

Os motivos alegados para a não realização do teste variaram, mas predominaram em mais da metade dos depoimentos, o fato de não achar necessário (34\%) e a falta de interesse (28\%). Outros depoimen- tos apontaram para o fato de não saber onde ele é ofertado ( $5 \%)$ e a inexistência dessa oferta ( $4 \%)$.

A distribuição das variáveis quanto à cor (tabela 5), pessoas classificadas como negras perceberam maior dificuldade no atendimento, maior relato de sinais e sintomas de DST, e maior frequência de automedicação quando comparadas as suas respostas às de pessoas não negras. Contudo, a avaliação do serviço foi considerada ótima/boa por parte de $45 \%$ delas. Entre pessoas não negras houve maior procura pelo serviço público para diagnóstico e tratamento de sintomas e sinais de DST, maior testagem para HIV e maior conhecimento sobre o local de realização do teste. 
Tabela 3 - Distribuição de variáveis relativas ao uso dos serviços de saúde para prevenção de DST/HIV/aids, segundo gênero em comunidades remanescentes de quilombos. Brasil, 2007-2008

\begin{tabular}{|c|c|c|c|c|c|}
\hline \multirow{2}{*}{ Variáveis } & & \multicolumn{2}{|c|}{ Gênero Masculino } & \multicolumn{2}{|c|}{ Gênero Feminino } \\
\hline & & $\mathrm{N}$ & $\%$ & $\mathrm{~N}$ & $\%$ \\
\hline \multirow{3}{*}{ Percepção de dificuldade de atendimento } & Sim & 45 & 49,5 & 52 & 45,6 \\
\hline & Não & 46 & 50,5 & 62 & 54,4 \\
\hline & Total & 91 & 100,0 & 114 & 100,0 \\
\hline \multirow{3}{*}{ DST autorreferida (últimos 6 meses) } & $\operatorname{sim}$ & 4 & 4,2 & 16 & 13,4 \\
\hline & Não & 91 & 95,8 & 103 & 86,6 \\
\hline & Total & 95 & 100,0 & 119 & 100,0 \\
\hline \multirow{7}{*}{ Conduta adotada diante da doença (DST) } & Serviço público & 3 & 60,0 & II & 68,8 \\
\hline & Farmácia & 1 & 20,0 & 0 & 0,0 \\
\hline & Automedicação & 0 & 0,0 & 0 & 0,0 \\
\hline & Serviço particular & 0 & 0,0 & 0 & 0,0 \\
\hline & Não fez nada & 0 & 0,0 & 2 & 12,5 \\
\hline & Outros & 1 & 20,0 & 3 & 18,8 \\
\hline & Total* & 5 & 100,0 & 16 & 100,0 \\
\hline \multirow{3}{*}{ Testagem para HIV } & $\operatorname{sim}$ & 16 & 16,7 & 31 & 25,6 \\
\hline & Não & 80 & 83,3 & 90 & 74,4 \\
\hline & Total & 96 & 100,0 & 121 & 100,0 \\
\hline \multirow{3}{*}{ Conhecimento sobre onde fazer o teste ${ }^{* *}$} & Sim & 58 & 72,5 & 57 & 67,1 \\
\hline & Não & 22 & 27,5 & 28 & 32,9 \\
\hline & Total & 80 & 100,0 & 85 & 100,0 \\
\hline \multirow{5}{*}{ Avaliação do serviço } & ótimo & 2 & 2,2 & 3 & 2,5 \\
\hline & Bom & 37 & 41,1 & 48 & 40,7 \\
\hline & Regular & 32 & 35,6 & 37 & 31,4 \\
\hline & Ruim & 19 & 21,1 & 30 & 25,4 \\
\hline & Total & 90 & 100,0 & 118 & 100,0 \\
\hline
\end{tabular}

* Totais não somam outras categorias incluídas menos frequentes.

** Calculado entre os participantes que não fizeram o teste. 
Tabela 4 - Distribuição de variáveis relativas ao uso dos serviços de saúde para prevenção de DST/HIV/aids, segundo gênero em comunidades remanescentes de quilombos. Brasil, 2007-2008

\begin{tabular}{|c|c|c|c|c|c|}
\hline \multirow{2}{*}{ Variáveis } & & \multicolumn{2}{|c|}{ Idade $<35$ anos } & \multicolumn{2}{|c|}{ Idade 35 e mais } \\
\hline & & $\mathrm{N}$ & $\%$ & $\mathrm{~N}$ & $\%$ \\
\hline \multirow{3}{*}{ Percepção de dificuldade de atendimento } & $\operatorname{sim}$ & 56 & 49,6 & 41 & 44,6 \\
\hline & Não & 57 & 50,4 & 51 & 55,4 \\
\hline & Total & 113 & 100,0 & 92 & 100,0 \\
\hline \multirow{3}{*}{ DST autorreferida (últimos 6 meses) } & $\operatorname{sim}$ & 12 & 10,3 & 8 & 8,2 \\
\hline & Não & 105 & 89,7 & 89 & 91,8 \\
\hline & Total & 117 & 100,0 & 97 & 100,0 \\
\hline \multirow{7}{*}{ Conduta adotada diante da doença (DST) } & Serviço público & 0 & 0,0 & 6 & 66,7 \\
\hline & Farmácia & 0 & 0,0 & 1 & 11,1 \\
\hline & Automedicação & 0 & 0,0 & 0 & 0,0 \\
\hline & Serviço particular & 8 & 66,7 & 0 & 0,0 \\
\hline & Não fez nada & 1 & 8,3 & 1 & 11,1 \\
\hline & Outros & 3 & 25,0 & 1 & 11,1 \\
\hline & Total* & 12 & 100,0 & 9 & 100,0 \\
\hline \multirow{3}{*}{ Testagem para HIV } & $\operatorname{sim}$ & 30 & 25,2 & 17 & 17,3 \\
\hline & Não & 89 & 74,8 & 81 & 82,7 \\
\hline & Total & 119 & 100,0 & 98 & 100,0 \\
\hline \multirow{3}{*}{ Conhecimento sobre onde fazer o teste ${ }^{* *}$} & $\operatorname{sim}$ & 56 & 65,9 & 59 & 73,8 \\
\hline & Não & 29 & 34,1 & 21 & 26,3 \\
\hline & Total & 85 & 100,0 & 80 & 100,0 \\
\hline \multirow{5}{*}{ Avaliação do serviço } & Ótimo & 3 & 2,6 & 2 & 2,2 \\
\hline & Bom & 45 & 39,1 & 40 & 43,0 \\
\hline & Regular & 41 & 35,7 & 28 & 30,1 \\
\hline & Ruim & 26 & 22,6 & 23 & 24,7 \\
\hline & Total & 115 & 100,0 & 93 & 100,0 \\
\hline
\end{tabular}

* Totais não somam outras categorias incluídas menos frequentes.

** Calculado entre os participantes que não fizeram o teste. 
Tabela 5 - Distribuição de variáveis relativas ao uso dos serviços de saúde para prevenção de DST/HIV/aids, segundo cor/raça em comunidades remanescentes de quilombos. Brasil, 2007-2008

\begin{tabular}{llcccc} 
Variáveis & & \multicolumn{2}{c}{ Cor/raça Negras } & \multicolumn{2}{c}{ Não negras } \\
Percepção de dificuldade de & Sim & $N$ & $\%$ & $N$ & $\%$ \\
atendimento & Não & 66 & 48,2 & 30 & 44,8 \\
& Total & 71 & 51,8 & 37 & 55,2 \\
\hline DST autorreferida (últimos 6 meses) & Sim & 137 & 100,0 & 67 & 100,0 \\
& Não & 14 & 9,9 & 6 & 8,5 \\
& Total & 128 & 90,1 & 65 & 91,5 \\
\hline Conduta adotada diante da doença & Serviço público & 142 & 100,0 & 71 & 100,0 \\
\hline (DST) & Farmácia & 0 & 0,0 & 3 & 50,0 \\
& Automedicação & 11 & 73,3 & 0 & 0,0 \\
& Serviço particular & 0 & 0,0 & 0 & 0,0 \\
& Não fez nada & 0 & 0,0 & 2 & 33,3 \\
& Outros & 3 & 20,0 & 1 & 16,7 \\
& Total* & 15 & 100,0 & 6 & 100,0 \\
\hline Testagem para HIV & Sim & 29 & 20,0 & 18 & 25,4 \\
& Não & 116 & 80,0 & 53 & 74,6 \\
& Total & 145 & 100,0 & 71 & 100,0 \\
\hline Conhecimento sobre onde fazer O & Sim & 72 & 64,9 & 42 & 79,2 \\
& Nẽo & 39 & 35,1 & 11 & 20,8 \\
& Total & 111 & 100,0 & 53 & 100,0 \\
\hline Avaliação do serviço & Ótimo & 4 & 2,9 & 1 & 1,4 \\
& Bom & 58 & 42,0 & 27 & 39,1 \\
& Regular & 45 & 32,6 & 23 & 33,3 \\
& Ruim & 31 & 22,5 & 18 & 26,1 \\
& Total & 138 & 100,0 & 69 & 100,0 \\
\hline
\end{tabular}

* Totais não somam outras categorias incluídas menos frequentes.

** Calculado entre os participantes que não fizeram o teste.

As razões alegadas para a avaliação ruim ou regular dos serviços foram muito parecidas com as explicações em relação às dificuldades encontradas para o acesso: deslocamentos, horários inconvenientes para pegar uma senha ou longas filas, implicando, ocasionalmente, a necessidade de pernoite. Além das razões ligadas à acessibilidade propriamente dita, foram mencionados outros fatores como: falta de realização de exames, falta de medicamentos, e, em geral, a não resolubilidade dos serviços. Este último aspecto foi particularmente precário em relação às demandas da saúde bucal.
Não. Ainda não, já marcamos várias vezes com as enfermeiras, mas nunca dá certo de vir. Nunca veio nada sobre orientação, não teve, aqui não. (Grupo focal masculino - Nova RomaMagalhães,GO.).

Ele morreu assim, sabe... ele não ganhou muito atendimento, entendeu? Ele não tinha condições e não ajudaram, isso que pareceu. Por isso que passou na TV. Muitas vezes você vai ao posto e não te atendem, com ele foi a mesma coisa. (Grupo focal masculino - Mormaça - Sertão, RS.). 
[Com relação ao teste para HIV na comunidade] Eu também só fiz quando estava grávida, antes de internar eles colheram meu sangue pra ver se eu não tinha. Se a gente quer fazer o exame não faz porque não tem no posto, só no hospital. (Grupo focal -feminino - Brejo dos Crioulos Varzelândia, MG.).

Pra marcar consulta é uma dificuldade, só tem médico uma vez por mês e a comunidade é grande, aífica ao Deus dará... (Grupo focal feminino - Baixo Ipixúna - Gurupá, PA.).

\section{Discussão}

Observou-se que nas comunidades em que o processo de discussão sobre remanescentes de quilombo foi desencadeado com maior participação dos moradores, a autorreferência da cor negra flui com naturalidade e orgulho. As comunidades mais organizadas têm mais facilidade de compreender as questões raciais do país. É interessante citar o trabalho de conscientização dos professores nas escolas em relação ao resgate das origens dessas comunidades.

É importante destacar que a maioria das comunidades quilombolas em que o trabalho foi realizado corresponde a pequenos coletivos rurais em que a agricultura é a principal fonte de sustento; $54 \%$ dos entrevistados nessas comunidades declararam como principal ocupação o trabalho rural. De maneira geral, observou-se que onde havia maior nível de organização, as condições de vida da coletividade eram melhores quando comparadas àquelas com nível de organização insipiente. 0 estudo apontou para condições sociais semelhantes às encontradas por outros pesquisadores (Lopes, 2003,). Vale a pena destacar que a mediana da renda declarada correspondeu a R\$ 200,oo, cifra inferior ao salário mínimo na época da realização da pesquisa (de 01/04/2007 a 01/03/2008, o salário mínimo oficial era de R\$ 380,00 , correspondente a cerca de US\$ 200,00 no câmbio desse mesmo período).

Em geral, há dificuldades concretas de acesso aos serviços. Quanto a sua oferta, a maioria das comunidades tem cobertura formal do PSF, no entanto, a população carece de assistência conforme relatos dos grupos focais. Em algumas áreas, como na de assistência odontológica, essa lacuna é particular- mente crítica, como referido pelos entrevistados de Tururu (CE). Isso remete a uma profunda discussão e reformulação das estratégias de oferta e organização de serviços no nível da Atenção Básica.

A análise do uso dos serviços de saúde por gênero revelou que as mulheres utilizam os serviços públicos mais do que os homens e são mais testadas para HIV nesses serviços. Essa diferença pode decorrer da oferta do teste na rotina do pré-natal. A maior frequência de relato de sinais e sintomas que poderiam corresponder a DST entre as mulheres pode ser por causa da maior captação pelo serviço. Essa questão remete ao fato da sensação de invulnerabilidade dos homens, à falta de autocuidados e à tradicional falta de uma política dirigida aos homens, que tem sido objeto de preocupação recente por parte do Ministério da Saúde (Braz, 2005).

A maior diferença constatada em relação ao uso de serviços segundo a idade consistiu na maior utilização do serviço público por parte de pessoas maiores de 35 anos. Esse fato contrasta com a maior utilização de serviços particulares por parte dos mais jovens. Essa assimetria pode ser explicada pelas diferentes percepções relacionadas à idade em relação às formas de exposição, além do estigma.

Quanto ao uso de serviços segundo a cor, a maior diferença nas proporções foi observada na conduta adotada diante de sinais e sintomas de DST. Pessoas classificadas como negras se automedicaram mais frequentemente do que pessoas não negras, entre as quais predominou a busca pelo serviço público. Essa diferença pode decorrer do estigma relacionado às DST, da inexistência do serviço e de tradições culturais.

Apesar do grande numero de pessoas que afirmaram saber o que é HIV/aids e como ele se transmite, uma série de mitos sobre a doença ainda é predominante no imaginário dessas populações, tais como a transmissão pela picada de insetos ou por banhar-se em rios ou lagos juntamente com pessoas portadoras do vírus. Não há dúvidas que a situação dessas comunidades não é ideal e não está próxima dela. Não podemos descartar, porém, os esforços nas últimas décadas, dos movimentos negros, das comunidades remanescentes de quilombo e do atual governo. Como já destacamos anteriormente, as respostas ao Decreto 4.887 , de 20 de novembro 
de 2003 (uma conquista desses movimentos), já são visíveis e trouxeram uma série de benefícios materiais e simbólicos para os quilombolas. Porém não se pode desguarnecer. 0 quadro atual só irá se modificar com políticas públicas de inclusão social que combatam o preconceito e deem acesso ao pleno direito de cidadania, que não se faz por lei, mas com educação de qualidade, saúde, acesso à informação e direitos e deveres, de fato, iguais.

Apesar de ter sido realizada uma amostragem aleatória dos locais da pesquisa, a amostra dos sujeitos quando contatados nos respectivos locais não foi probabilística, limitando a generalização das prevalências obtidas para o universo da população estudada (Pereira, 1999). Outra limitação decorre do tipo de estudo (transversal), em que há vieses de prevalência/sobrevivência e ambiguidade temporal. Além disso, a formulação de perguntas sobre temas sensíveis pode levar a constrangimentos incorrendo em vieses de aferição/informação. Essas possibilidades foram minimizadas pelo treino dos entrevistadores e pela formulação cuidadosa das questões.

Os achados deste estudo reafirmam a situação de vulnerabilidade da população negra na questão do acesso e da utilização dos serviços de saúde. O estudo reafirma a necessidade de políticas públicas específicas voltadas aos segmentos mais vulneráveis, levando-se em conta a tendência da epidemia. É importante ressaltar a necessidade de capacitação e incentivo às equipes dos programas PACS e PSF, haja vista sua relevância na assistência dessas comunidades.

\section{Referências}

AYRES, J. R. C. M. et al. O conceito de vulnerabilidade e as práticas de saúde: novas perspectivas e desafios. In: CZERESNIA, D.; FREITAS, C. M. (Org.). Promoção da Saúde: conceitos, reflexões, tendências. Rio de Janeiro: Fiocruz, 2003. p. 119-139.

BRAZ, M. A construção da subjetividade masculina e seu impacto sobre a saúde do homem: reflexão bioética sobre justiça distributiva. Ciência \& Saúde Coletiva (versão online), Rio de Janeiro, v. 10, n. 1, jan./mar. 2005. Disponível em: < http://www.scielosp.org/scielo.php?pid=S1413$81232005000100016 \&$ script=sci_arttext $>$. Acesso em 21 abr. 2009.
IBGE - Instituto Brasileiro de Geografia e Estatística. Censo Demográfico 20oo: Características da População e dos Domicílios: Resultados do universo. Disponível em: < http:// www.ibge.gov.br/home/estatistica/populacao/ censo20oo/default.shtm>. Acesso em 21 de abril 2009.

GOMES, F. S. A hidra e os pântanos: mocambos, quilombos e comunidades de fugitivos no Brasil (Séculos XVII-XIX). São Paulo: Unesp; Polis, 2005.

LOPES, F. Mulheres negras e não negras vivendo com HIV/aids no estado de São Paulo: um estudo sobre suas vulnerabilidades. 2003. $203 \mathrm{f}$. Tese (Doutorado em Saúde Pública) - Departamento de Epidemiologia, Faculdade de Saúde Pública da Universidade de São Paulo, São Paulo, 2003.

MANN, J.; TARANTOLA, D. J. M. From epidemiology to vulnerability, to human rights. In: MANN, J.; TARANTOLA, D. J. M. (Orgs.). AIDS in the World II. Global Dimensions, Social Roots and Responses. New York: Oxford University Press, 1996. p. 427462.

BRASIL. Ministério da Saúde. A Saúde da População Negra e o SUS - Ações Afirmativas para Avançar na Equidade. Brasília: Ministério da Saúde, 2005. (Série B, Textos Básicos em Saúde).

BRASIL. Ministério da Saúde. Governo lança Programa contra aids dirigido à população negra - 15/o8/2005. Disponível em: < http://www. jornalcco.com.br/site/index.php?basePrincipal $=\mathrm{m}$ ateriasVisualizar\&materia $=3726>$. Acesso em: 20 jan. 2009 .

PEREIRA, M. G. Epidemiologia: teoria e prática. Rio de Janeiro: Koogan, 1996.

SIMÃO, A. B.; MIRANDA-RIBEIRO, P.; CAETANO, A. J. Raça/Cor e uso de serviços de atendimento ginecológico em Belo Horizonte em 2002: uma análise exploratória. Belo Horizonte: UFMG, 2007.

Recebido em: 07/10/2009

Reapresentado em: 22/04/2010

Aprovado em: 26/04/2010 